\title{
LA FAMILIA PENTATOMIDAE (HEMIPTERA: HETEROPTERA) EN LAS COLECCIONES DE REFERENCIA DE LA REPÚBLICA DOMINICANA
}

\author{
Gabriel de los Santos ${ }^{1}$ y Ruth Bastardo ${ }^{2}$ \\ ${ }^{1}$ Museo Nacional de Historia Natural "Prof. Eugenio de Jesús Marcano". \\ Calle César Nicolás Penson, Plaza de la Cultura Juan Pablo Duarte, \\ Santo Domingo, República Dominicana.g.delossantos@mnhn.gov.do \\ ${ }^{2}$ Instituto de Investigaciones Botánicas y Zoológicas Prof. Rafael M. Moscoso, Universidad Autónoma \\ de Santo Domingo, Santo Domingo, República Dominicana.r_bastardo@hotmail.com
}

\begin{abstract}
RESUMEN
Se describen las colecciones de la familia Pentatomidae(Hemiptera: Heteroptera) depositadas en el Museo Nacional de Historia Natural "Prof. Eugenio de Jesús Marcano" (MNHNSD) y en el Instituto de Investigaciones Botánicas y Zoológicas Rafael Ma. Moscoso (IIBZ) de la Universidad Autónoma de Santo Domingo. Se encontraron 2,113 especímenes entre ambas colecciones, distribuidos en 31 géneros y 60 especies, los cuales representan, respectivamente, 86 y $76 \%$ de los géneros y especies registrados para La Hispaniola. La colección del MNHNSD contiene 1,189 especímenes, distribuidos en 29 géneros y 57 especies, mientras que la del IIBZ posee 924 especímenes, distribuidos en 28 géneros y 49 especies. Ocho especies endémicas de La Hispaniola están representadas en estas colecciones. No se registró ningún espécimen colectado en Haití. Pharypia pulchella (Drury), es tratada como una especie de presencia dudosa debido a que se encontró un único espécimen sin datos de colecta.
\end{abstract}

Palabras clave: Pentatomidae, colección de referencia, República Dominicana, La Hispaniola.

Title: The family Pentatomidae (Hemiptera: Heteroptera) in the reference collections of the Dominican Republic.

\section{ABSTRACT}

The collections of the Pentatomidae family (Hemiptera: Heteroptera) housed at the Museo Nacional de Historia Natural "Prof. Eugenio de Jesús Marcano" (MNHNSD) and the Instituto de Investigaciones Botánicas y Zoológicas Prof. Rafael Ma. Moscoso (IIBZ) at the Universidad Autónoma de Santo Domingo are described. 2,113 specimens were found among both collections, distributed in 31 genera and 60 species, representing 86 and 76\%, of the genera and species reported from Hispaniola. The collection at the MNHNSD holds 1,189 specimens, distributed in 29 genera and 57 species, while the one at the IIBZ holds 924 specimens, distributed in 28 genera and 49 species. Eight Hispaniolan endemic species are represented in these collections. No specimen collected in Haiti was found. Pharypia pulchella (Drury) is treated as a species of doubtful presence because just an unlabeled specimen was found.

Key words: Pentatomidae, reference collection, Dominican Republic, Hispaniola.

\section{INTRODUCCIÓN}

A nivel mundial, esta familia posee alrededor de 4,700 especies descritas, comprendidas en unos 900 géneros (Rider, 2006a). Los pentatómidos tienen gran importancia económica y agrícola, llegando en algunos casos a ser plagas de cultivos. Por otro lado, algunos de sus miembros (subfamilia Asopinae) se alimentan de otros insectos, reconociéndoseles su potencial 
como controles biológicos (Ortega-León, 1997; Costello et al, 2002; Pérez-Gelabert y Thomas, 2005). En República Dominicana son conocidos con el nombre de "hiede vivos".

La tesis en Ingeniería Agronómica de Rodríguez (1980), sobre la biología de Nezara viridula (Linnaeus), es el primer trabajo realizado por un dominicano con la familia Pentatomidae. Posteriormente, Reynoso (1982, 1984, 1985a y 1985b) trata la morfología y la biología de varias especies de pentatómidos. Thomas (1994), describe el género Mediocampus para la República Dominicana. El trabajo más amplio sobre esta familia en La Hispaniola es el de Pérez-Gelabert y Thomas (2005), en el que se catalogan 77 especies y se describen siete nuevas para la ciencia. Actualmente, se reconocen 79 especies para la isla, 31 para Haití y 75 para República Dominicana (28 compartidas); 14 de estas especies son endémicas, la mayoría de ellas en la República Dominicana (12), incluyendo dos géneros (Thomas, 1994; Pérez-Gelabert y Thomas, 2005; McPherson y Ahmad, 2011; De los Santos y Bastardo, 2012).

En la República Dominicana existen dos importantes colecciones de insectos. La colección depositada en el Museo Nacional de Historia Natural "Prof. Eugenio de Jesús Marcano" (MNHNSD), es la más grande que tiene el país (más de 90,000 especímenes); mientras que la depositada en el Instituto de Investigaciones Botánicas y Zoológicas Prof. Rafael Ma. Moscoso (IIBZ) de la Universidad Autónoma de Santo Domingo, es la más antigua (iniciada en el año 1958 por el profesor Eugenio de Js. Marcano).

En este trabajo se describe el estatus de la familia Pentatomidae en las colecciones de referencia de la República Dominicana, así como también se presentan datos sobre distribución de las especies, los colectores más importantes e imágenes de algunas de ellas. Los resultados aquí presentados son parte de la tesis de Licenciatura en Biología del primer autor.

\section{OBJETIVO}

- Dar a conocer el estatus de la familia Pentatomidae en las colecciones de referencia de la República Dominicana.

\section{MATERIALES Y MÉTODOS}

Los especímenes fueron curados usando alcohol al 95\%. En el caso de la colección del IIBZ, se hizo necesaria la determinación de los especímenes, la elaboración de nuevas etiquetas con los datos de colecta y el montaje de algunos de ellos.

Las determinaciones se hicieron comparando con especímenes de referencia identificados por Donald B. Thomas, así como utilizando claves y dibujos de genitalias ofrecidos en revisiones de diferentes géneros (i.e., Brailovsky, 1981; Eger, 1978 y 1980; Grazia, 1980; Rider y Chapin, 1992; Rolston, 1978, 1983 y 1986; Sailer, 1944; Thomas y Yonke, 1988). El ordenamiento sistemático se hizo siguiendo a Pérez-Gelabert y Thomas (2005).

Las imágenes fueron tomadas utilizando un microscopio estereoscópico marca Leica, modelo MZ7.5, el cual tiene acercamientos entre $0.63 \mathrm{x}$ y $5.0 \mathrm{x}$, y al que se le adaptaron objetivos de $0.5 \mathrm{x}$ y $1.0 \mathrm{x}$, según la necesidad, y una cámara Nikon modelo Coolpix 4500; otra cámara Nikon modelo D1x, con un lente AF Micro Nikkor 105mm 1:2.8 D, se utilizó para las especies de mayor tamaño. Las imágenes se procesaron utilizando el programa Auto Montage, versión 5.0, y se editaron con el programa Adobe PhotoShop, versión 7.0.

En los totales de especies se incluyen morfos determinados solamente a nivel de género: Mediocampus sp., Mormidea sp., Edessa sp. 1 y Edessa sp. 2 (ver Anexo). En cambio, no se cuentan los morfos sin determinación de género. 
La distribución de las especies endémicas Banasa flavosa Thomas, Mediocampus dominicanus Thomas, Mediocampus perezi Thomas, Mediocampus woodruffi Thomas y Edessa rawlinsi Thomas fue tomada de Pérez-Gelabert y Thomas (2005).

\section{RESULTADOS}

Un total de 2,113 especímenes están depositados en ambas colecciones, distribuidos en 31 géneros y 60 especies; hay 46 especies en común, tres solo en el IIBZ y 11 solo en el MNHNSD (Tablas 1 y 2). Hay 1,189 especímenes, 29 géneros y 57 especies en el MNHNSD, mientras que la colección del IIBZ incluye 924 ejemplares, 28 géneros y 49 especies (Tabla 1). Se hizo la determinación específica de 1,718 especímenes (801 en el IIBZ, 917 en el MNHNSD); de estos, 198 (74 en el IIBZ y 124 en el MNHNSD) solo pudieron ser determinados hasta el nivel de género. A otros dos especímenes (IIBZ) no se les pudo determinar el género. No se encontró ningún espécimen colectado en Haití.

En cuanto a la representatividad, en la colección del IIBZ se encuentra el 62\% de las especies registradas para La Hispaniola, mientras que en el MNHNSD se encuentra el $72 \%$. Entre ambas poseen el $76 \%$ de las especies registradas para la isla (Tabla 2).

Los géneros mejor representados, tomando en cuenta solamente aquellos con más de una especie, son Acrosternum Fieber (5 especies), Cyptocephala Berg (3), Fecelia Stål (3), Podisus Herrich-Schäffer (5) y Thyanta Stål (3), con el 100\% de las especies registradas. Las especies endémicas con especímenes en estas colecciones fueron Acrosternum (Chinavia) insulani Rolston (Fig. 1), Antillosciocoris palisoti Thomas, Banasa punctata Thomas, Fecelia biorbis Eger (Fig. 14), Murgantia thomasi McPherson et Ahmad, Oebalus magnus Thomas, Stiretrus quinquepunctatus Germar (Fig. 19) y Mediocampus sp. (esta última incluida por tratarse de un género endémico).

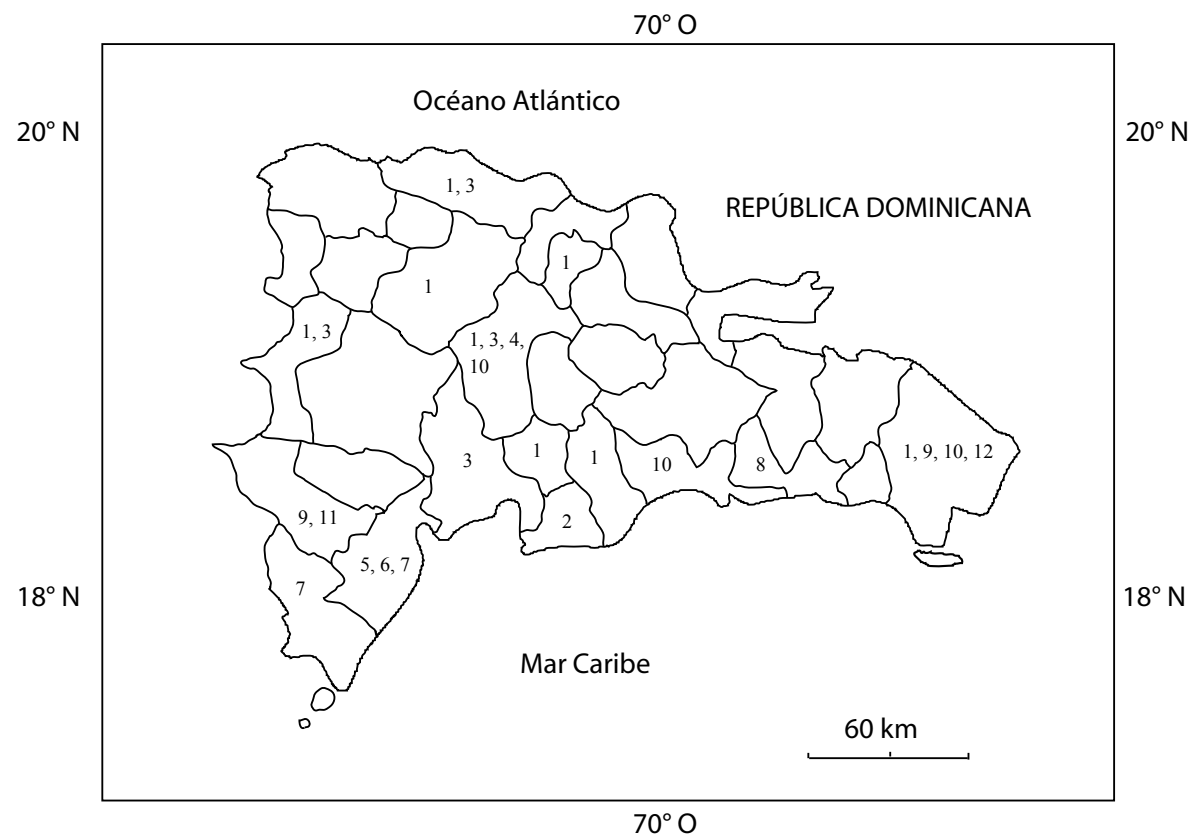

Figura 1. Mapa de distribución de las especies endémicas de La Hispaniola que se encuentran en la República Dominicana. Acrosternum (Chinavia) insulani Rolston (1); Antillosciocoris palisoti Thomas (2); Banasa flavosa Thomas (3); Banasa punctata Thomas (4); Edessa rawlinsi Thomas (5); Fecelia biorbis Eger (6); Mediocampus dominicanus Thomas (7); Mediocampus perezi Thomas (8); Mediocampus woodruffi Thomas (9); Murgantia thomasi McPherson et Ahmad (10); Oebalus magnus Thomas (11); Stiretrus quinquepunctatus Germar (12). 
Las especies Thyanta (Thyanta) perditor (Fabricius), Fig. 7; Euschistus bifibulus (Beauvois), Fig. 2 y E. crenator (Fabricius), Fig. 3, fueron las más abundantes en la colección del IIBZ (Tabla 1). En el MNHNSD fueron N. viridula, Fig. 5; Oebalus pugnax (Fabricius), Fig. 6 y Loxa viridis (Beauvois), Fig. 4. De manera general, las especies mejor representadas en estas dos colecciones son $T$. (Thyanta) perditor, $N$. viridula, E. crenator, E. bifibulus y L. viridis.

Tabla 1. Cantidad de especímenes por especie en cada colección.

\begin{tabular}{|c|c|c|c|}
\hline \multirow{2}{*}{ ESPECIE } & \multicolumn{2}{|c|}{ COLECCIÓN } & \multirow[b]{2}{*}{ Total } \\
\hline & IIBZ & MNHNSD & \\
\hline Alcaeorrynchus phymatophorus & 6 & 9 & 15 \\
\hline Andrallus spinidens & 5 & - & 5 \\
\hline Podisus maculiventris & 4 & 2 & 6 \\
\hline Podisus mucronatus & 1 & 5 & 6 \\
\hline Podisus sagitta & 24 & 15 & 39 \\
\hline Podisus subferrugenius & 1 & 1 & 2 \\
\hline Stiretrus quinquepunctatus & - & 1 & 1 \\
\hline Tylospilus accutissimus & 8 & 8 & 16 \\
\hline Acrosternum (Chinavia) insulani & 6 & 9 & 15 \\
\hline Acrosternum (Chinavia) marginatum & 30 & 49 & 79 \\
\hline Acrosternum (Chinavia) montivagum & 1 & 2 & 3 \\
\hline Acrosternum (Chinavia) ubicum & 4 & 1 & 5 \\
\hline Acrosternum (Chinavia) wygodzinskyi & 8 & 53 & 61 \\
\hline Acrosternum spp. & 17 & 16 & 33 \\
\hline Agonoscelis puberula & 6 & 2 & 8 \\
\hline Arocera (Euopta) placens & 5 & 4 & 9 \\
\hline Arvelius albopunctatus & - & 2 & 2 \\
\hline Arvelius sp. & 16 & 39 & 55 \\
\hline Banasa herbacea & 11 & 7 & 18 \\
\hline Banasa punctata & - & 1 & 1 \\
\hline Banasa punctatissima & - & 12 & 12 \\
\hline Banasa zeteki & 8 & 56 & 64 \\
\hline Banasa sp. & 6 & 14 & 20 \\
\hline Caribo fasciatus & 7 & 8 & 15 \\
\hline Cyptocephala antiguensis & 27 & 44 & 71 \\
\hline Cyptocephala bimini & - & 1 & 1 \\
\hline Cyptocephala pallida & 24 & 15 & 39 \\
\hline Cyptocephala sp. & 8 & 18 & 26 \\
\hline Euschistus acuminatus & 11 & 6 & 17 \\
\hline Euschistus bifibulus & 75 & 45 & 120 \\
\hline Euschistus crenator & 58 & 71 & 129 \\
\hline Euschistus obscurus & 1 & 2 & 3 \\
\hline
\end{tabular}


Tabla 1 (continuación).

\begin{tabular}{|c|c|c|c|}
\hline \multirow{2}{*}{ ESPECIE } & \multicolumn{2}{|c|}{ COLECCIÓN } & \multirow[b]{2}{*}{ Total } \\
\hline & IIBZ & MNHNSD & \\
\hline Euschistus sp. & 1 & 6 & 7 \\
\hline Fecelia biorbis & 7 & - & 7 \\
\hline Fecelia nigridens & 11 & 18 & 29 \\
\hline Fecelia proxima & 1 & 8 & 9 \\
\hline Fecelia sp. & 1 & - & 1 \\
\hline Grazia tincta & 2 & - & 2 \\
\hline Loxa pallida & - & 17 & 17 \\
\hline Loxa viridis & 34 & 74 & 108 \\
\hline Loxa sp. & 2 & 1 & 3 \\
\hline Mediocampus sp. & 1 & 4 & 5 \\
\hline Mormidea albisignis & 4 & 4 & 8 \\
\hline Mormidea cubrosa & 44 & 38 & 82 \\
\hline Mormidea sp. & 8 & 1 & 9 \\
\hline Murgantia sp. & 8 & 7 & 15 \\
\hline Nezara viridula & 55 & 87 & 142 \\
\hline Oebalus linki & 6 & 1 & 7 \\
\hline Oebalus magnus & 6 & 10 & 16 \\
\hline Oebalus ornatus & 52 & 26 & 78 \\
\hline Oebalus pugnax & 27 & 78 & 105 \\
\hline Oebalus ypsilongriseus & 23 & 40 & 63 \\
\hline Piezodorus guildinii & 38 & 11 & 49 \\
\hline Proxys victor & 38 & 11 & 49 \\
\hline Thyanta (Argosoma) obsoleta & 1 & 2 & 3 \\
\hline Thyanta (Argosoma) testacea & - & 3 & 3 \\
\hline Thyanta (Thyanta) perditor & 86 & 60 & 146 \\
\hline Thyanta sp. & 15 & 12 & 27 \\
\hline Tibraca limbativentris & 2 & 6 & 8 \\
\hline Vulsirea nigrorubra & - & 4 & 4 \\
\hline Brepholoxa rotundifrons & 7 & 16 & 23 \\
\hline Brepholoxa sp. & - & 10 & 10 \\
\hline Mecidea longula & 18 & 38 & 56 \\
\hline Antillosciocoris palisoti & - & 1 & 1 \\
\hline Edessa bifida & 29 & 52 & 81 \\
\hline Edessa meditabunda & 15 & 19 & 34 \\
\hline Edessa sp. 1 & - & 1 & 1 \\
\hline Edessa sp. 2 & - & 2 & 2 \\
\hline Piezosternum subulatum & 2 & 3 & 5 \\
\hline Indeterminada & 2 & - & 2 \\
\hline
\end{tabular}


Tabla 2. Representatividad de las colecciones con respecto a los registros en La Hispaniola.

\begin{tabular}{|c|c|c|c|c|}
\hline & Géneros & Especies & $\begin{array}{c}\text { Porcentaje de representación } \\
\text { de géneros }\end{array}$ & $\begin{array}{c}\text { Porcentaje de representación } \\
\text { de especies }\end{array}$ \\
\hline La Hispaniola & 36 & 79 & - & - \\
\hline MNHNSD & 29 & 57 & 81 & 72 \\
\hline IIBZ & 28 & 49 & 78 & 62 \\
\hline Ambas colecciones & 31 & 60 & 86 & 76 \\
\hline
\end{tabular}

Se encontraron varias especies consideradas de importancia económica, Acrosternum (Chinavia) marginatum (Palisot de Beauvois), Arvelius albopunctatus (De Geer), Fig. 8; Cyptocephala antiguensis (Westwood), Edessa meditabunda (Fabricius), Fig. 9; Euschistus bifibulus, E. crenator, Mormidea cubrosa (Dallas), Oebalus ornatus (Sailer), Fig. 10; O. pugnax (Fabricius), O. ypsilongriseus (De Geer), Fig. 11; Piezodorus guildinii (Westwood), Fig. 12; Tibraca limbativentris Stål, Fig. 13 y Thyanta (Thyanta) perditor.

En lo que a distribución respecta, Euschistus crenator es la especie con el rango más amplio, habiéndose colectado en 25 provincias (Tabla 3). Le siguen Acrosternum (Chinavia) marginatum (Beauvois), Euschistus bifibulus, N. viridula y Edessa bifida (Say), colectadas en 21 provincias cada una; Cyptocephala antiguensis (Westwood), L. viridis y T. (Thyanta) perditor en 20 provincias cada una. Mientras que Podisus subferrugineus Barber et Bruner, Stiretrus quinquepunctatus, Fecelia biorbis, Vulsirea nigrorubra Spinola y Antillosciocoris palisoti se conocen de una sola provincia. De $A$. palisoti, se ha colectado un solo espécimen en la provincia Peravia.

En cuanto a la representatividad de especies por provincia, Pedernales es donde se ha colectado la mayor cantidad, con 41 (Tabla 3); seguida de La Vega (34), Barahona (33), La Altagracia (32) y Santo Domingo (31). A pesar de que todas las provincias están representadas en las colecciones, algunas presentan poca representatividad, con apenas siete especies cada una: Bahoruco, Dajabón, María Trinidad Sánchez y San Pedro de Macorís.

Se encontró que los meses con mayor cantidad de registros y especies son: abril (147 registros y 44 especies), julio (141 y 43), junio (131 y 38), diciembre (116 y 37) y agosto (113 y 37), Tabla 4. Febrero y septiembre son los meses con menos registros (47 y 48, respectivamente); mientras que, en cuanto a la cantidad de especies, febrero, marzo y enero (20, 21 y 22, respectivamente), poseen las menores cantidades.

El hallazgo de las especies Oebalus linki (Heidemann), Piezosternum subulatum (Thunberg) y Podisus subferrugineus (Figs. 15, 16 y 18, respectivamente) fue tratado recientemente por De los Santos y Bastardo (2012). Las dos primeras representan nuevos registros para República Dominicana y La Hispaniola, mientras que $P$. subulatum representa un nuevo hallazgo luego de casi 200 años sin registros (Pérez-Gelabert y Thomas, 2005: 347).

Los registros más antiguos en el IIBZ son los de L. viridis y $N$. viridula, ambos en la provincia La Vega, en fecha 23.XII.1958 y VIII.1958, respectivamente; mientras que en el MNHNSD, el más antiguo corresponde a Piezodorus guildinii (Westwood), de fecha 10.XII.1969, también en la provincia La Vega. Por otro lado, los colectores que han hecho los mayores aportes a estas colecciones son, Héctor Domínguez, Eugenio de Js. Marcano, Daniel Pérez-Gelabert, Ruth Bastardo, Brígido Hierro, Kelvin Guerrero, Félix Del Monte y Blas Reynoso. Además, se encontró que Donald B. Thomas y David A. Rider, especialistas de esta familia, han contribuido en la determinación de muchos de los especímenes de estas colecciones. 


\begin{tabular}{|c|c|c|c|c|c|c|c|c|c|c|c|c|c|c|c|c|c|c|c|c|c|c|c|c|c|c|c|c|c|c|}
\hline$\nexists$ & $\infty$ & $\mathrm{N}$ in & in & $\simeq$ & -- & $-\infty$ & $=$ & $\vec{\sim}$ & in & $\theta$ & $=$ & $=$ & + & + & $+=$ & $y 0$ & 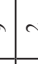 & r & $=$ & $r$ & $a$ & ¿ & $m r$ & $a$ & $=$ & & $\therefore$ & & & $\simeq n$ \\
\hline के & $x$ & & & $x$ & & & & $x$ & & $x$ & $x$ & $x$ & $x$ & & $y$ & $4 x$ & & & & $x$ & & $x$ & & & $x$ & $x$ & 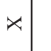 & & & $x$ \\
\hline 8 & & $\not$ & 4 & $x$ & & $x$ & $4 x$ & 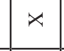 & & & & & $x$ & & $>$ & 4 & & & $x$ & & & $x$ & & & & $x$ & $x$ & & & \\
\hline$\sum$ & & & & $x$ & & & & 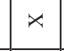 & & & $x$ & $x$ & & & & & & & & & & & & & & $x$ & $x$ & & & $\approx$ \\
\hline$\hat{z}$ & & & & $x$ & & & & $x$ & & & & & & & & & & & & & & $\rtimes$ & & $x$ & & & $\rtimes$ & & & \\
\hline$\frac{z}{z}$ & & & & $x$ & & & & $x$ & & & & $x$ & & & & & & & $\approx$ & $x$ & & & & & & $x$ & $x$ & & & $\star$ \\
\hline$\$$ & & & & & & & & & & & & & & & & & & & & & & $x$ & $x$ & $x$ & $x$ & $x$ & 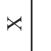 & & & \\
\hline 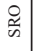 & & & & & & $x$ & & & & & & & & & & & & & & & & $x$ & & $x$ & $\approx$ & & $x$ & $x$ & & \\
\hline 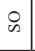 & & $\approx$ & $\varepsilon$ & & & $x$ & $x$ & $x$ & $x$ & & $\approx$ & $x$ & & & $y$ & $4 x$ & & & & & $\approx$ & $x$ & & & & & $\rtimes$ & & & $\star$ \\
\hline$\approx$ & & & & $x$ & & & $x$ & & & & & & & & & & & & $x$ & & & & & & & & $x$ & & & \\
\hline is & & & & & & & & & & & $\approx$ & & & & & 8 & E & & & & & & & & & $x$ & $x$ & & & \\
\hline $\bar{c}$ & & & & $x$ & & & & $x$ & & & $\approx$ & & & & & & & & $\approx$ & & & $x$ & & & & & $x$ & & & $x$ \\
\hline 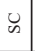 & & $x$ & & $x$ & & & $x$ & $x$ & & & $x$ & & & & $>$ & 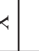 & & & $x$ & $x$ & $x$ & 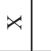 & & & & $x$ & $\rtimes$ & & & \\
\hline$\overleftarrow{\Delta}$ & & & & & & & & $\approx$ & & $x$ & 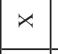 & & & & 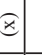 & & & & & & & $x$ & & & & $x$ & & & & \\
\hline 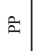 & $x$ & & & & & & $x$ & $x$ & & & $x$ & $x$ & & & $>$ & 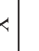 & & $x$ & $x$ & & & $x$ & & & & $x$ & $x$ & & & 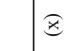 \\
\hline 2 & & & $x$ & $x$ & & $x$ & & $x$ & & $x$ & $x$ & & & & & $x$ & 4 & & & & & $x$ & $x$ & $x$ & $x$ & & $x$ & & & $x$ \\
\hline 罢 & $\widehat{\approx}$ & & $\approx$ & $x$ & & & $x$ & $x$ & $\widehat{\approx}$ & & $x$ & $x$ & & $\otimes$ & $\widehat{\otimes}>$ & $x$ & & $x$ & $\approx$ & $x$ & $x$ & & $x \times$ & $4 x$ & $x$ & $x$ & $x>$ & $x$ & & $x$ \\
\hline$\Sigma$ & & & & & & $x$ & & & & & & & & & & & & $x$ & & & $x$ & $\times 3$ & $\otimes x$ & $x$ & & & & & & \\
\hline$\Sigma$ & & & & & & & & $x$ & & $\approx$ & & & & & & & & & & & & & & & & $x$ & & & & \\
\hline 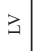 & $x$ & $x$ & 4 & $x$ & $\star$ & $\approx$ & $x$ & $x$ & & & & & $\approx>$ & $x$ & $y$ & $4 x$ & $<x$ & & $x$ & $x$ & $\widehat{\star}$ & $x$ & $x$ & $<$ & $\approx$ & $x$ & 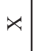 & & & $x \mid x$ \\
\hline$\dddot{\exists}$ & & & & & & & & & & & $x$ & $x$ & & & & & & & & & & & & & $x$ & & $x>$ & $x$ & & \\
\hline$\Xi$ & 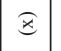 & & $x$ & $x$ & & $x$ & $\approx$ & $\times$ & & & $x$ & $x$ & & & $\otimes>$ & $x$ & & & & $x$ & $x$ & $x$ & & & & $x$ & $\star x$ & $*$ & & $\rtimes$ \\
\hline$z$ & & & & $\widehat{\otimes}$ & & & & $x$ & $\approx$ & & $\times$ & $x$ & & & & & & & & & $x$ & $x 3$ & $\otimes x$ & $<x$ & $x$ & & $x$ & & & $\rtimes \approx$ \\
\hline$\underline{\underline{\mathbf{z}}}$ & $x$ & & & & & & 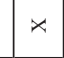 & & & & 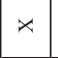 & $x$ & & & & & & & & & & & & & $x$ & $x$ & & & & \\
\hline$\underline{4}$ & & & & $\widehat{\Xi}$ & & & & $x$ & & & & & & & $>$ & 4 & & & & & & $x$ & & & & $x$ & $x$ & & & \\
\hline 出 & & & & $x$ & & & & $x$ & & & & & $\widehat{\star}$ & & $>$ & 4 & & & & & & $x$ & & & & $x$ & $x$ & & & \\
\hline 产 & $x$ & & & $x$ & & & $\approx$ & $x$ & 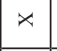 & & $x$ & & & & & & & $\approx$ & $\approx$ & & $\approx$ & & & & $x$ & $x$ & $x$ & & & $x$ \\
\hline ฉ & & & & & & & & & $\approx$ & & & & & & & & & $x$ & & & & $x$ & & & & $x$ & & & & \\
\hline$\vec{a}$ & & $x$ & & & & & & & & & & & & & & & & & & & & & & & & & $\rtimes$ & & & \\
\hline$\approx$ & & $\approx$ & $\varepsilon$ & $\approx$ & & & $\approx$ & $x$ & & & $x$ & $x$ & & $\star \triangleright$ & $x>$ & $x$ & $4 \times$ & $x$ & $x$ & $x$ & & $x$ & & $x$ & & $x$ & $x$ & & & $\approx \approx$ \\
\hline 품 & & $x$ & $x$ & & & $x$ & & & & & & & & & & & & & & & & & $\approx$ & $\varepsilon$ & & & & & & \\
\hline y & $x$ & & $x$ & $x$ & & $x$ & & $x$ & & & $x$ & & & $x$ & $>$ & 43 & $\varepsilon$ & $x$ & $\approx$ & & $x$ & $x$ & & $x$ & $x$ & & $x$ & & & $x$ \\
\hline & 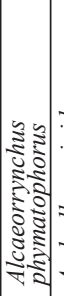 & 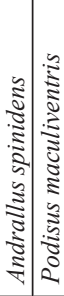 & 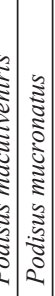 & 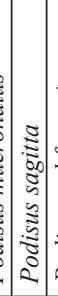 & 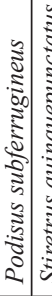 & 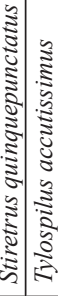 & 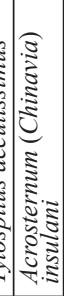 & 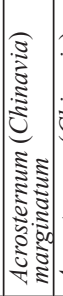 & 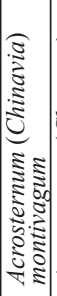 & 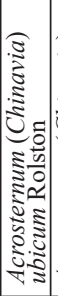 & 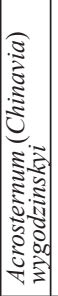 & 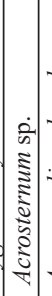 & 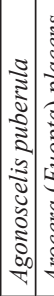 & 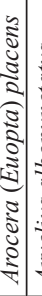 & 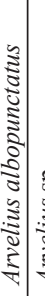 & 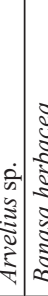 & 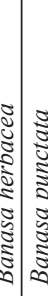 & 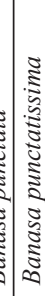 & 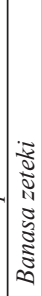 & 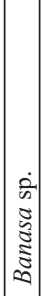 & 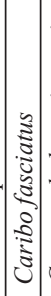 & 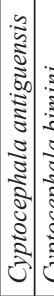 & 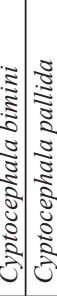 & 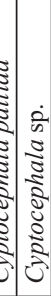 & 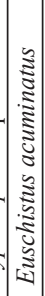 & 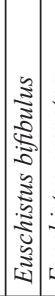 & 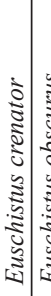 & 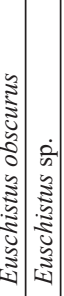 & 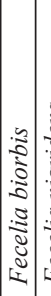 & 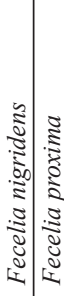 \\
\hline
\end{tabular}




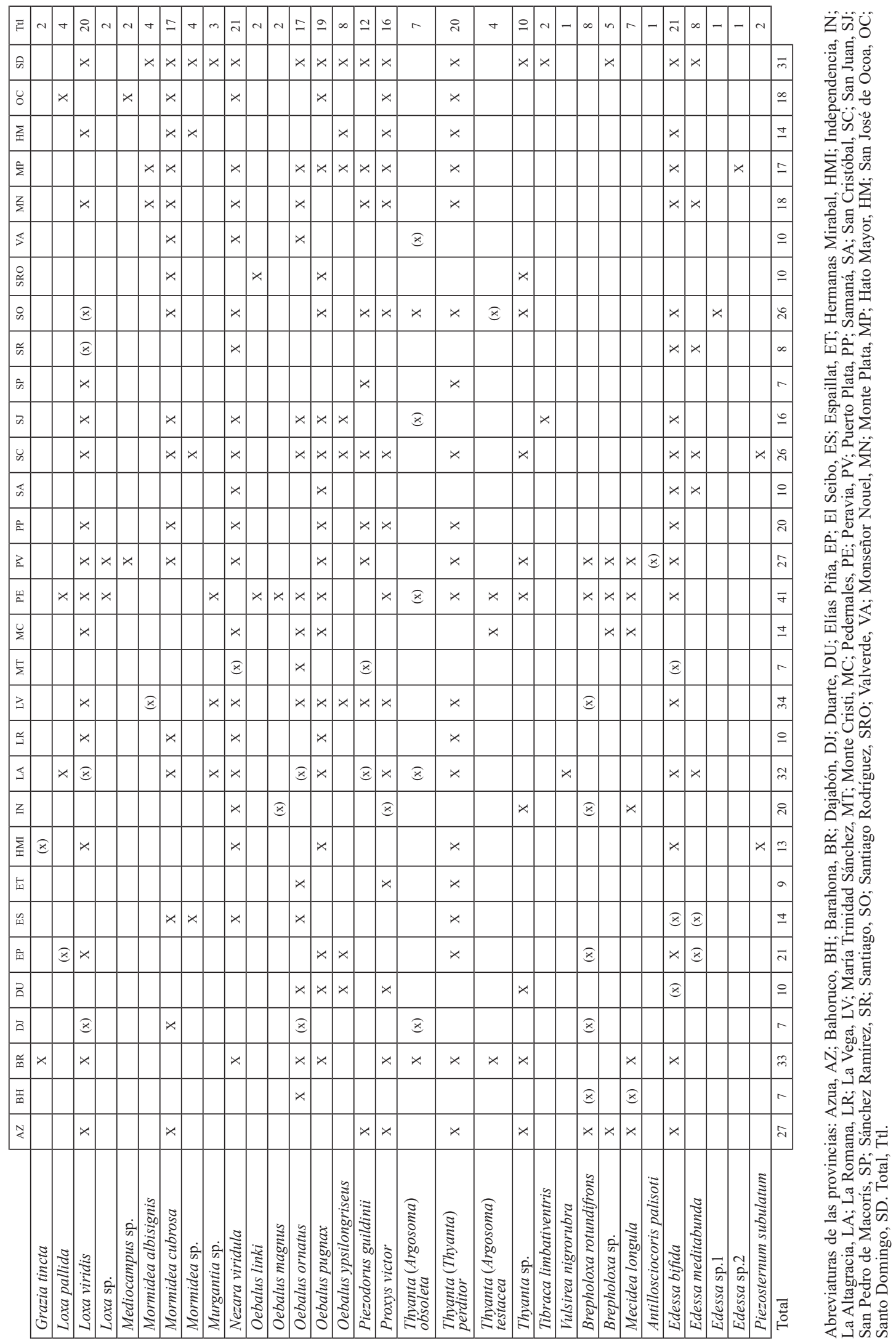



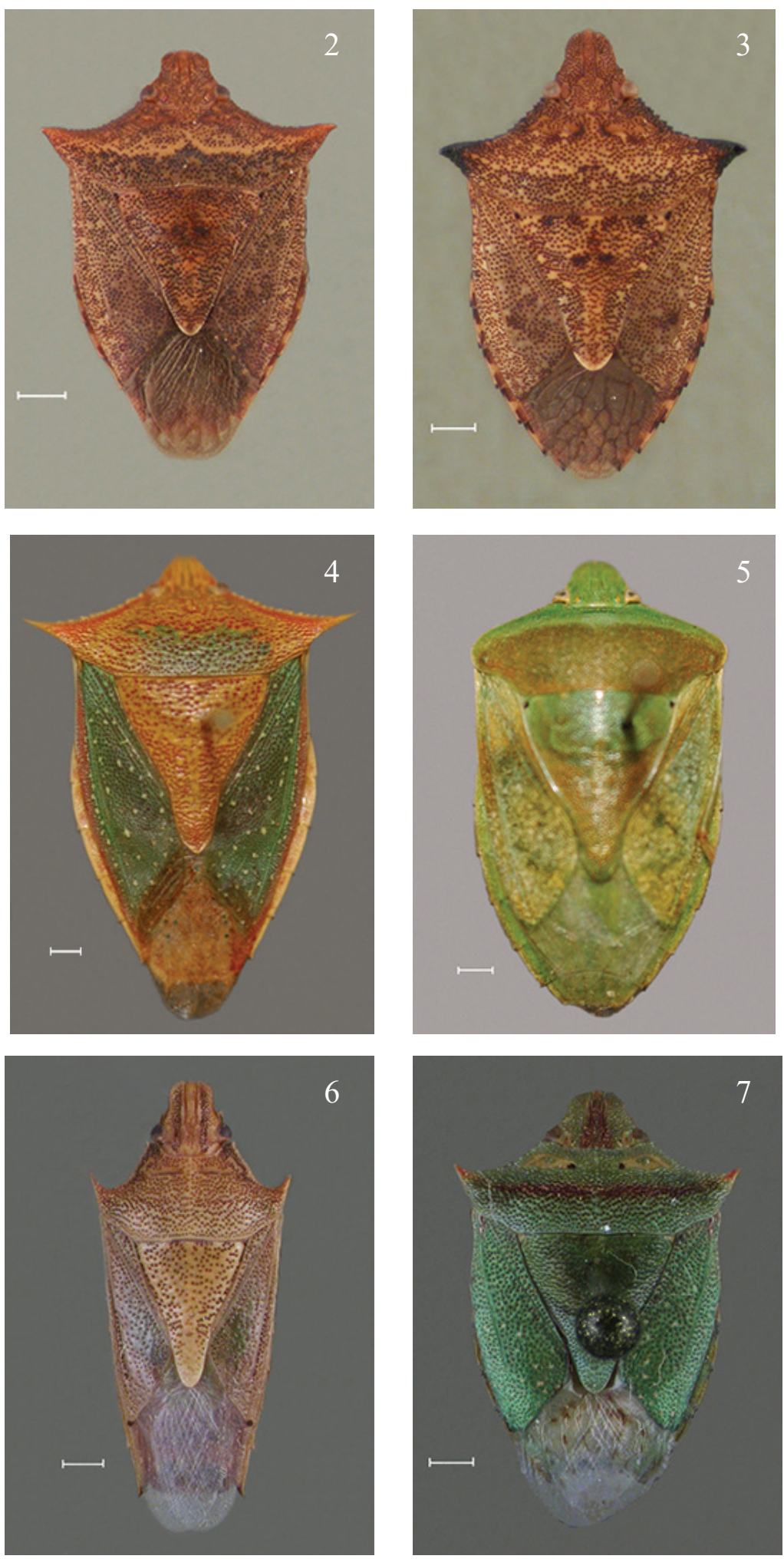

Figuras 2-7. Especies más comunes en las colecciones. 2, Euschistus bifibulus (Beauvois); 3, Euschistus crenator (Fabricius); 4, Loxa viridis (Palisot de Beauvois); 5, Nezara viridula (Linnaeus); 6, Oebalus pugnax (Fabricius); 7, Thyanta (Thyanta) perditor (Fabricius). Escala: $1.0 \mathrm{~mm}$. 

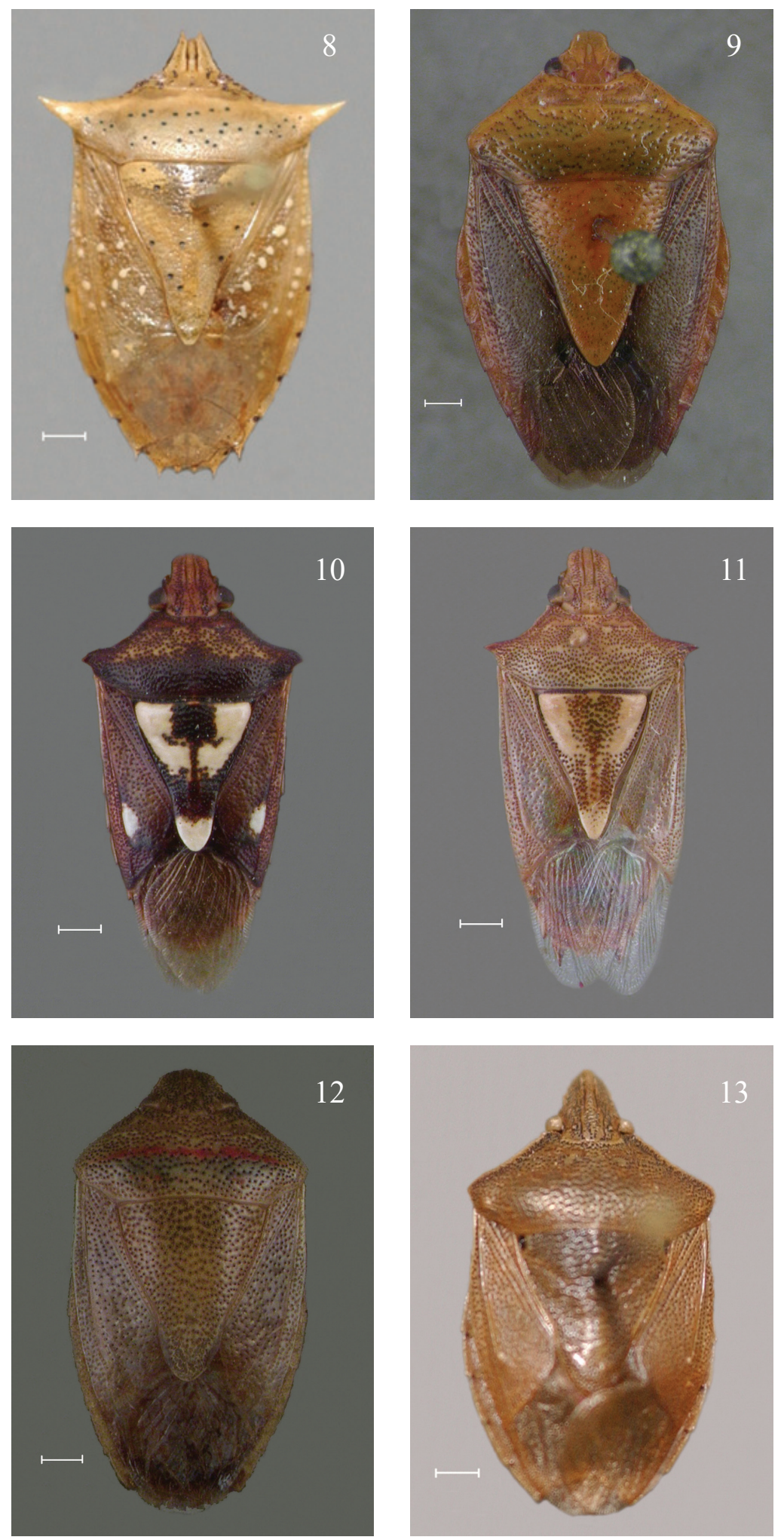

Figuras 8-13. Especies con importancia económica. 8, Arvelius albopunctatus (De Geer); 9, Edessa meditabunda (Fabricius); 10, Oebalus ornatus (Sailer); 11, O. ypsilongriseus (De Geer); 12, Piezodorus guildinii (Westwood); 13, Tibraca limbativentris Stål. Escala: $1.0 \mathrm{~mm}$. 

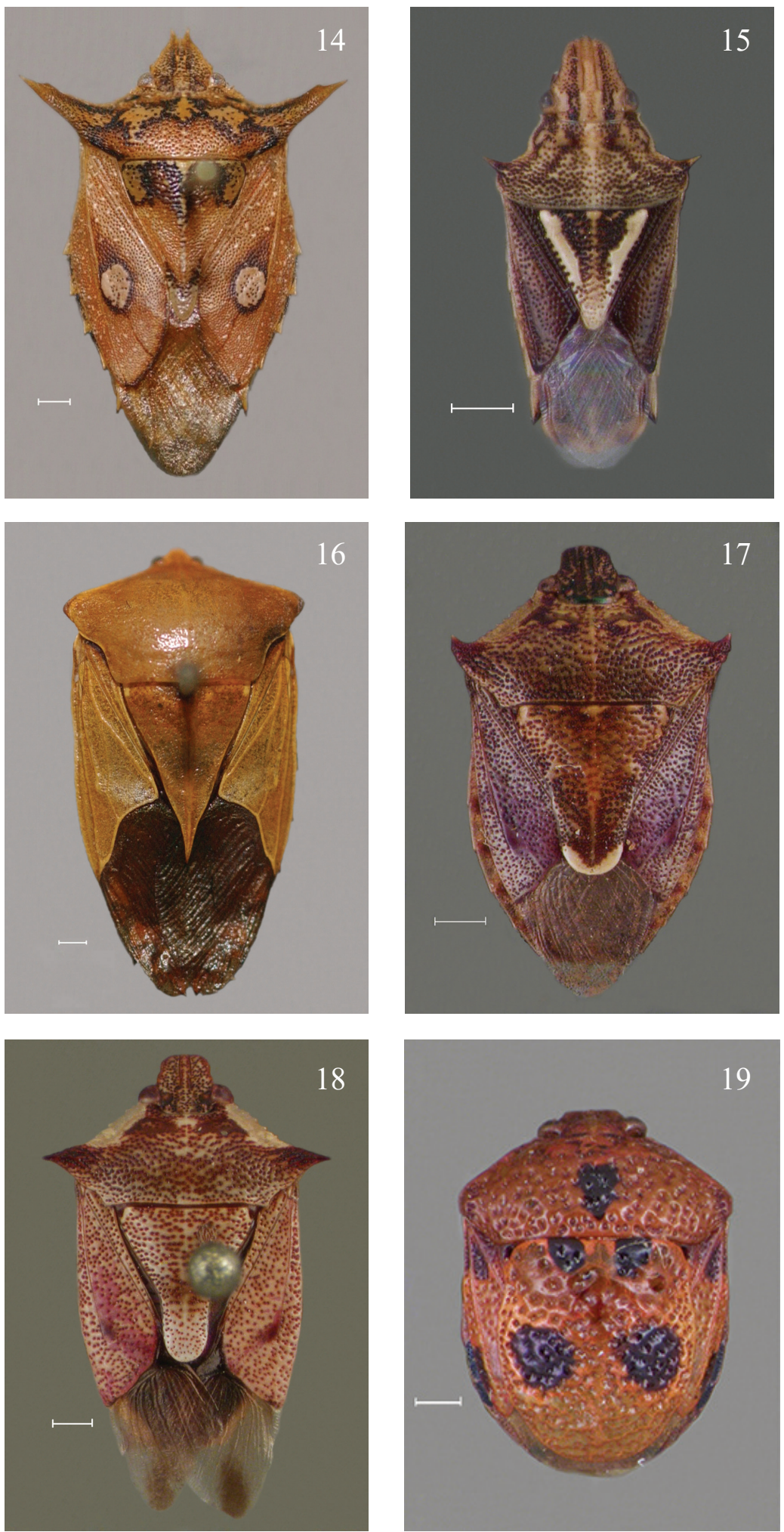

Figuras 14-19. Especies registradas recientemente. 14, Fecelia biorbis Eger; 15, Oebalus linki (Heidemann); 16, Piezosternum subulatum (Thunberg); 17, Podisus mucronatus Uhler; 18, Podisus subferrugineus Barber et Bruner; 19, Stiretrus quinquepunctatus Germar. Escala: $1.0 \mathrm{~mm}$. 
Tabla 4. Cantidad de registros y especies colectadas en cada mes.

\begin{tabular}{|c|c|c|c|c|c|c|c|c|c|c|c|c|}
\cline { 2 - 12 } \multicolumn{1}{c|}{} & Ene & Feb & Mar & Abr & May & Jun & Jul & Ago & Sep & Oct & Nov & Dic \\
\hline Total de registros & 52 & 47 & 59 & 147 & 98 & 131 & 141 & 113 & 48 & 50 & 71 & 116 \\
\hline Total de especies & 22 & 20 & 21 & 44 & 34 & 38 & 43 & 37 & 25 & 27 & 28 & 37 \\
\hline
\end{tabular}

\section{DISCUSIÓN}

Los valores de representatividad obtenidos para ambas colecciones no deberán ser considerados como definitivos, toda vez que aún queda un apreciable número de especímenes sin determinar. Estos morfos que siguen sin identificar podrían tratarse de táxones nuevos para la ciencia.

El hecho de que la provincia Pedernales sea la única que registra más de 40 especies, se explica por el mayor número de expediciones de colecta realizadas hacia esta zona, reconocida por su elevada diversidad biológica, incluyendo dos áreas protegidas de gran tamaño: Parque Nacional Jaragua y Parque Nacional Sierra de Bahoruco. Esta tendencia a priorizar las colectas en la región suroeste ha dejado como resultado un pobre conocimiento sobre la diversidad de los pentatómidos en unas 19 provincias del país (i.e., asumiendo 20 especies como una buena representación).

Algo parecido ocurre con los meses de colecta, siendo preferencia de los entomólogos extranjeros y nacionales realizar las expediciones en primavera y verano, principalmente en el período junio-agosto. Durante los meses más fríos y secos, enero-marzo, se han realizado menos expediciones de colecta, ocurriendo lo mismo con el periodo septiembre-noviembre. El valor elevado registrado en abril tiene su razón en expediciones realizadas por el MNHNSD durante las décadas de 1970 y 1990 y en un amplio estudio de la entomofauna de la isla durante el período 2002-2004. Al margen de estas consideraciones, las especies E. bifibulus, E. crenator, L. viridis, N. viridula, Mormidea cubrosa (Dallas), Oebalus pugnax, Piezodorus guildinii, Podisus sagitta (Fabricius) y T. (Thyanta) perditor son las más abundantes.

Por la falta de etiqueta con datos de colecta en el ejemplar de Pharypia pulchella (Drury), se sugiere agregarla a la lista de especies con dudosa presencia en la isla ofrecida por PérezGelabert y Thomas (2005: 347-348). En esa lista, los autores referidos incluyen a Antiteuchus piceus (Beauvois), Arvelius crassispinus Breddin, Edessa rufomarginata (De Geer) y Euschistus ictericus (Linnaeus). Después de la revisión hecha por los autores citados y el presente trabajo, incluyendo siete colecciones y aproximadamente 4,000 especímenes, se apuntala la convicción de que estas especies no se encuentran en la isla, aunque quizá no sea el mismo caso de $P$. pulchella. Además de esto, en las Antillas todavía no se tienen registros de estas especies, con excepción, otra vez, de $P$. pulchella, la que se conoce de Puerto Rico (Pérez-Gelabert, en preparación). En el caso particular de $A$. crassispinus, se recomienda consultar las consideraciones de PérezGelabert y Thomas (2005: 348) y Rider (2006b), quienes proponen que $A$. crassispinus, debe ser una sinonimia de Arvelius albopunctatus (De Geer).

\section{AGRADECIMIENTOS}

Especial agradecimiento a Donald B. Thomas (USDA-ARS, Cattle Fever Tick Research Laboratory), por su valiosa y desinteresada ayuda en nuestras consultas sobre taxonomía y distribución de las especies, además de la literatura facilitada. A Brian Farrell (MCZ, Harvard 
University) por darme la oportunidad de formar parte del acuerdo UASD - Universidad de Harvard, al que este trabajo se le incluye como parte de sus resultados. A Thomas J. Henry del U. S. National Museum of Natural History, Smithsonian Institution (USNM), por las facilidades brindadas con la colección de dicho museo; a Daniel Pérez-Gelabert (USNM) por su ayuda con la literatura y todas sus sugerencias. También a Manuel Valdez (IIBZ) y Celeste Mir (MNHNSD), por las facilidades ofrecidas para la revisión de las colecciones. A Carlos Suriel (MNHNSD), por sus sugerencias.

\section{LITERATURA CITADA}

Brailovsky, H. 1981. Revisión del género Arvelius Spinola (Hemiptera-HeteropteraPentatomidae-Pentatomini). Anales del Instituto de Biología Universidad Nacional Autónoma de México, Serie Zoología, 51 (1): 239-298.

Costello, S. L., P. D. Pratt, M. B. Rayachhetry y T. D. Center. 2002. Morphology and life history characteristics of Podisus mucronatus (Heteroptera: Pentatomidae). Florida Entomologist, 85 (2): 344-350.

De los Santos, G. y R. Bastardo. 2012. Nuevos registros de hiedevivos (Insecta: Hemiptera: Pentatomidae) para la República Dominicana y La Hispaniola, incluyendo el nuevo hallazgo de Piezosternum subulatum (Thunberg, 1783). Novitates Caribeae, 4: 120-123.

Eger, J. E., Jr. 1978. Revision of the genus Loxa (Hemiptera: Pentatomidae). Journal of the New York Entomological Society, 86: 224-259.

Eger, J. E., Jr. 1980. Fecelia biorbis n. sp. (Heteroptera: Pentatomidae), a new species from Haiti. Journal of the New York Entomological Society, 88: 29-32.

Grazia, J. 1980. Uma nova especie do género Fecelia Stål (Heteroptera, Pentatomidae, Pentatomini). Revista Brasileira de Biologia, 40 (2): 261-266.

McPherson, J. E., y I. Ahmad. 2011. Murgantia thomasi, a new species of stink bug from the Dominican Republic (Hemiptera: Heteroptera: Pentatomidae). Annals of the Entomological Society of America, 104 (2): 149-153.

Ortega-León, G. 1997. Distribución de la subfamilia Asopinae (Hemiptera: Heteroptera: Pentatomidae) para México. Anales del Instituto de Biología Universidad Nacional Autónoma de México, Serie Zoología, 68: 53-89.

Pérez-Gelabert, D. E. 2008. Arthropods of Hispaniola (Dominican Republic and Haiti): A checklist and bibliography. Zootaxa, 1831: 1-530.

Pérez-Gelabert, D. E., y D. B. Thomas. 2005. Stink bugs (Pentatomidae: Heteroptera) of the island of Hispaniola, with seven new species from the Dominican Republic. Boletín de la Sociedad Entomológica Aragonesa, 37: 319-352.

Reynoso S., B. 1982. Estudio sobre el Desarrollo y Morfología de los Insectos Oebalus ornata Sailer y Oebalus insularis Stål Hemiptera - Pentatomidae. Tesis de Licenciatura en Biología, Universidad Autónoma de Santo Domingo, Santo Domingo, 97 p. [Sin publicar]

Reynoso S., B. 1984. Desarrollo y morfología del Thyanta perditor Fabricius (HemipteraPentatomidae). Memoria de la 2da Jornada Científica Medio Ambiente y Recursos Naturales, Santo Domingo, pp. 173-174. [Resumen] 
Reynoso S., B. 1985a. Morfología y biología (ciclo del huevo al adulto) del Nezara viridula Linn. (Hemiptera-Pentatomidae). Resúmenes I Congreso Dominicano de Zoología, MNHN, Santo Domingo, Dic. 1985. [Sin paginar]

Reynoso S., B. 1985b. Morfología y biología (ciclo del huevo al adulto) del Oebalus pugnax Fabr. (Hemiptera-Pentatomidae). Resúmenes I Congreso Dominicano de Zoología, MNHN, Santo Domingo, Dic. 1985. [Sin paginar]

Rider, D. 2006a. Number of Genera \& Species, Pentatomoidea Home Page. http://www.ndsu. nodak.edu/ndsu/rider/Pentatomoidea/Classification/Genus_Species_Numbers.htm. (22 de noviembre, 2012).

Rider, D. 2006b. Number of Genera \& Species, Pentatomoidea Home Page. http://www.ndsu. nodak.edu/ndsu/rider/Pentatomoidea/Genus_Chlorocorini/Arvelius.htm. (22 de noviembre, 2012).

Rider, D. A., y J. B. Chapin. 1992. Revision of the genus Thyanta Stål, 1862 (Heteroptera: Pentatomidae) II. North America, Central America, and the West Indies. Journal of the New York Entomological Society, 100: 42-98.

Rodríguez R., P. D. A. 1980. Estudio biológico del Nezara viridula (L.) (Hemiptera: Pentatomidae). Tesis de Ingeniería Agronómica, Universidad Autónoma de Santo Domingo, Santo Domingo, 45 p. [Sin publicar]

Rolston, L. H. 1978. A revision of the genus Mormidea (Hemiptera: Pentatomidae). Journal of the New York Entomological Society, 86: 161-219.

Rolston, L. H. 1983. A revision of the genus Acrosternum Fieber, subgenus Chinavia Orian, in the western hemisphere (Hemiptera: Pentatomidae). Journal of the New York Entomological Society, 91: 97-176.

Rolston, L. H. 1986. The genus Cyptocephala Berg, 1883 (Hemiptera: Pentatomidae). Journal of the New York Entomological Society, 94: 424-433.

Sailer, R. I. 1944. The genus Solubea (Heteroptera: Pentatomidae). Proceedings of the Entomological Society of Washington, 46: 105-127.

Thomas, D. B. 1992. Taxonomic synopsis of the Asopine Pentatomidae (Heteroptera) of the Western Hemisphere. Thomas Say Monographs No. 16, Entomological Society of America, Lanham, Maryland. 156 pp.

Thomas, D. B. 1994. Mediocampus, a new stinkbug genus from the Dominican Republic (Insecta: Heteroptera: Pentatomidae). Annals of the Carnegie Museum, 63: 257-261.

Thomas, D. B., y T. R. Yonke. 1988. Review of the genus Banasa Stål 1860 (Hemiptera: Pentatomidae) for Mexico, Central America and the Antilles. Annals of the Entomological Society of America, 81: 28-49.

[Recibido: 15 de marzo, 2013. Aceptado para publicación: 06 de agosto, 2013] 
Anexo. Lista de las especies encontradas en las colecciones.

Subfamilia ASOPINAE

Alcaeorrhynchus Bergroth, 1891

1. A. phymatophorus (Palisot de Beauvois, 1812)

Andrallus Bergroth, 1905

2. A. spinidens (Fabricius 1787)

Podisus Herrich-Schäffer, 1851

3. P. maculiventris (Say, 1831)

4. P. mucronatus Uhler, 1897

5. P. sagitta (Fabricius, 1794)

6. P. subferrugineus Barber et Bruner, 1932

Stiretrus Laporte, 1833

7. S. quinquepunctatus Germar, 1839*

Tylospilus Stål, 1870

8. T. accutissimus (Stål, 1870)

Subfamilia PENTATOMINAE

Tribu Pentatomini

Acrosternum Fieber, 1860

9. A. (Chinavia) insulani Rolston, 1983*

10. A. (Chinavia) marginatum (Palisot de Beauvois, 1805)

11. A. (Chinavia) montivagum (Distant, 1890)

12. A. (Chinavia) ubicum Rolston, 1983

13. A. (Chinavia) wygodzinskyi Rolston, 1983

Acrosternum sp.

Agonoscelis Spinola, 1837

14. A. puberula Stål, 1857

Arocera Spinola, 1837

15. A. (Euopta) placens (Walker, 1867)

Arvelius Spinola, 1837

16. A. albopunctatus (De Geer, 1773)

Arvelius sp.

Banasa Stål, 1860

17. B. herbacea (Stål, 1872)

18. B. punctata Thomas, 2005*

19. B. punctatissima Barber et Bruner, 1932

20. B. zeteki Sailer, 1959

Banasa sp.

Caribo Rolston, 1984

21. C. fasciatus Rolston, 1984

Cyptocephala Berg, 1883

22. C. antiguensis (Westwood, 1837)

23. C. bimini (Ruckes, 1952)

24. C. pallida Rolston, 1986

Cyptocephala $\mathrm{sp}$.

Euschistus Dallas, 1851

25. E. acuminatus Walker, 1867

26. E. bifibulus (Palisot de Beauvois, 1805)

27. E. crenator (Fabricius, 1794)

28. E. obscurus (Palisot de Beauvois, 1817)

Euschistus sp.

Fecelia Stål, 1872

29. F. biorbis Eger, 1980*

30. F. nigridens (Walker, 1867)

31. F. proxima Grazia, 1980

Fecelia sp.
Grazia Rolston, 1980

32. G. tincta (Distant, 1880)

Loxa Amyot et Serville, 1843

33. L. pallida Van Duzee, 1907

34. L. viridis (Palisot de Beauvois, 1805)

Loxa sp.

Mediocampus Thomas, 1994*

35. Mediocampus sp.*

Mormidea Amyot et Serville, 1843

36. M. albisignis Stål, 1872

37. M. cubrosa (Dallas, 1851)

38. Mormidea sp.

Murgantia Stål, 1862

39. M. thomasi McPherson et Ahmad, 2011*

Nezara Amyot et Serville, 1843

40. N. viridula (Linnaeus, 1758)

Oebalus Stål, 1862

41. O. linki (Heidemann, 1917)

42. O. magnus Thomas, 2005*

43. O. ornatus (Sailer, 1944)

44. O. pugnax (Fabricius, 1775)

45. O. ypsilongriseus (De Geer, 1773)

Piezodorus Fieber, 1860

46. P. guildinii (Westwood, 1837)

Proxys Spinola, 1837

47. P. victor (Fabricius, 1775)

Thyanta Stål, 1862

48. T. (Argosoma) obsoleta (Dallas, 1851)

49. T. (Argosoma) testacea (Dallas, 1851)

50. T. (Thyanta) perditor (Fabricius, 1794)

Thyanta sp.

Tibraca Stål, 1860

51. T. limbativentris Stål, 1860

Vulsirea Spinola, 1837

52. V. nigrorubra Spinola, 1837

Tribu Procletini

Brepholoxa Van Duzee, 1904

53. B. rotundifrons Barber, 1939

Brepholoxa sp.

Tribu Mecidiini

Mecidea Dallas, 1851

54. M. longula Stål, 1854

Tribu Sciocorini

Antillosciocoris Thomas, 2005*

55. A. palisoti Thomas, 2005*

Subfamilia EDESSINAE

Edessa Fabricius, 1803

56. E. bifida (Say, 1832)

57. E. meditabunda (Fabricius, 1794)

58. Edessa sp. 1

59. Edessa sp. 2 Subfamilia TESSARATOMINAE

Piezosternum Amyot et Serville, 1843

60. P. subulatum (Thunberg, 1783)

*endémica de La Hispaniola. 